\title{
MISZELLE
}

DIETRICH SCHUBERT

\section{NIETZSCHE UND SEINE EINWIRKUNGEN \\ IN DIE BILDENDE KUNST - EIN DESIDERAT HEUTIGER KUNSTGESCHICHTSWISSENSCHAFT?}

\author{
"- die Wissenschaft unter der Optik \\ des Künstlers zu sehen, die Kunst aber \\ unter der des Lebens...."
}

(GT, Versuch einer Selbstkritik 2)

Wie es um die Aufarbeitung und das Fragen nach der Nietzsche-Wirkung und Nietzsche-Rezeption in der bildenden Kunst nach 1890 im Fach der Kunsthistorik ${ }^{1}$ heute steht, beleuchtet keine Tatsache besser, als daß der letzte Beitrag zu Nietzsches Kunst-Verständnis - als dem einer ,gegenseitigen Notwendigkeit“ des Dionysischen und des Apollinischen - nicht von einem Kunstwissenschaftler veröffentlicht wurde, sondern von einem Philosophen, freilich von einem, dem die wesentlichen Werke der Kunstgeschichte auch Paradigmen der Geschichte des Geistes sind, Dieter Jähnig. ${ }^{2}$ Sein Aufsatz über Nietzsches Kunstbegriff (erläutert an der Geburt der Tragödie) erschien 1972.

Zwei weitere Beispiele: Nietzsche als präfaschistischen Philosophen, als Kämpfer für den wilhelminischen Imperialismus, als typischen Philosophen der „Großmannssucht" (P. Cassirer, W. Bode) des Wilhelminismus und als geistigen Wegbereiter und Vorläufer des Nazitums sehen Richard Hamann und Jost Hermand im Teil 1 ihrer „Epochen der deutschen Kultur", dem 1965 in Ost-Berlin und 1971 in München erschienenen Band über die Gründerzeit. ${ }^{3}$

Nietzsche überhaupt nicht genannt ist in dem jüngsten Buch über Rudolf Steiner ${ }^{4}$. Dabei war Steiner erster Archivar im Nietzsche-Archiv Weimar, natürlich von

${ }^{1} \mathrm{Zu}$ den Begriffen Kunsthistorik - Kunstwissenschaft - Kunstgeschichtswissenschaft, die hier nicht eigens unterschieden werden sollen, vgl. L. Coellen, Uber die Methode der Kunstgeschichte, Darmstadt 1924.

2 Dieter Jähnig, Nietzsches Kunstbegriff, in: Beiträge zur Theorie der Künste im 19. Jh., Bd. 2, Frankfurt/M. 1972, 29-68 und ders., Welt-Geschichte: Kunst-Geschichte, Köln 1975.

3 R. Hamann/Jost Hermand, Gründerzeit, Berlin-Ost 1965, München 1971

${ }_{4}^{4}$ Walter Kugler, Rudolf Steiner und die Anthroposophie - Wege zu einem neuen Menschen, Köln 1978 
Nietzsches Lebensphilosophie so oder so beeinflußt und verfaßte schon 1895 ein Buch über Nietzsche, den großen "Flammenwerfer" - wie Benn Nietzsche einmal nannte. ${ }^{5}$

Könnte man Nietzsche demnach - in Abwandlung eines Ausdrucks von Hegel und unter Verwendung eines typischen Begriffs der DDR-Geschichtsschreibung - eine ,Leiche im Keller der Erbe-Rezeption der Kunsthistorik nennen? Und ist er etwa noch nicht entnazifiziert?

Hier muß unterschieden werden: In der DDR ist Nietzsche eine Unperson, wird in den Buchhandlungen unterbunden, gilt - wie ihn Franz Mehring und G. Lukács einschätzten ${ }^{6}$ - als Philosoph des Imperialismus Wilhelms II. und ist tatsächlich auch noch nicht entnazifiziert.

Im Westen hat - außerhalb der Philosophie - die Literaturhistorik seit Jahren die enorme Bedeutung Nietzsches für die Ideengeschichte und die dichterischen Konkretionen um 1900, im Symbolismus und besonders im Expressionismus erkannt und befindet sich mitten in der Aufarbeitung der Einflüsse.

Doch was die Kunstgeschichtswissenschaft anbelangt, so scheint es, daß immer noch eine derart umfassende Abstinenz gegenüber der Wirkung des Entlarvungsphilosophen besteht, die unerklärlich anmutet, besonders in der älteren Generation der Kunstwissenschaftler. Liegt diese Abstinenz an der Indienstnahme durch die Nazis und einer damit verbundenen ängstlichen Scheu vor Nietzsche? Oder liegt sie an der Ambivalenz der Philosophie Nietzsches selbst? - wohl kaum!

Kurt Badt, der als Angehöriger der Expressionisten-Generation und als jüdischer Emigrant genügend Grund hatte, gegenüber den Faschisten und z. B. auch dem Freiburger Ordinarius der Philosophie, Martin Heidegger, mehr als Skepsis zu hegen, schrieb 1960 seinen kunsttheoretischen Beitrag „Feiern durch Rühmung“, wo Nietzsche nach Kant, Schelling, Hegel und Schopenhauer und vor Burckhardt, Conrad Fiedler und Heidegger seinen ihm gebührenden Platz erhielt. ${ }^{7}$

Jedenfalls hat man in der Kunsthistorik versäumt, beizeiten sich ein NietzscheBild $\mathrm{zu}$ erarbeiten, das von dem bei Lukács, Hamann/Hermand abweicht und Nietzsche gerecht werden kann. Hamann/Hermand zitieren beispielsweise fortlaufend den Macht-Historiker Treitschke und den Philosophen Nietzsche mit dem gleichen Beweisziel. Für Nietzsche war aber ,Macht' gerade nicht diejenige, die ,gereift, gerüstet und entschlossen . . . bei der neuen Teilung des Erd-Balles" im Sinne des jungen Kaisers ist ${ }^{8}$, sondern war sie vielmehr ein geistiger Begriff, bezogen auf eine Elite Edler und Schaffender (Künstler). Nietzsche würde, ,wie Flaubert, die Herrschaft einer

5 Rudolf Steiner, Nietzsche - ein Kämpfer gegen seine Zẹi, Weimar 1895, Neuaufl. Dornach 1926; - Gottfried Benn, Nietzsche nach fünfzig Jahren, in: Benn - Gesammelte Werke, hg. von D. Wellershoff, Wiesbaden, Bd. I, 1959, 482.

${ }^{6}$ Franz Mehring, Nietzsche (1898), jetzt in: Mehring - Werkauswahl Band III. hg. von Fritz J. Raddatz, Neuwied 1975, 118 f. - Georg Lukács, Die Zerstörung der Vernunft, Berlin 1955, $244 \mathrm{f}$. ,Nietzsche als Begründer des Irrationalismus der imperialistischen Periode“.

7 Kurt Badt. Feiern durch Rühmung, in: Kunsttheoretische Versuche, hg. von L. Dittmann, Köln 1968, $114 \mathrm{f}$.

8 So lautet eine Formulierung über Peter Behrens', ,Vorhalle zum Haus der Macht und der Schönheit" (Turin 1902) von G. Fuchs (in: Deutsche Kunst u. Dekoration XI, 1902/03, S. 6) 
Akademie verlangt haben, anstatt eines Klüngels von Waffenfabrikanten und Generalen" (Heinrich Mann). Den geistigen Charakter des Machtbegriffs Nietzsches hat schon 1919 Heinrich Mann gültig erläutert in „Kaiserreich und Republik“ .9 Jenes Zitieren von Treitschke und Nietzsche nebeneinander als vermeintlich gleiche Zeugen des wilhelminischen Wahns bei Hamann/Hermand geschah übrigens durchaus im Bewußtsein um die prinzipiellen Aussagen Nietzsches über den Staat als dem kältesten aller kalten Ungeheuer: „Dort, wo der Staat aufhört, da beginnt erst der Mensch, der nicht überflüssig ist" ${ }^{\text {"10 }}$, schrieb Nietzsche im, von den Expressionisten als programmatisch empfundenen Zaratbustra. Wie absurd ein gleichatmiges Anrufen von Treitschke und Nietzsche als Zeugen des Wilhelminismus ist, wird einem erst klar, wenn man Nietzsches Aussage über Treitschke kennt! Er kommt in Ecce bomo auf den ,Fall Wagner', dessen Antisemitismus ihn abstieß, zu sprechen und umschreibt sogleich seine Stellung zu den Deutschen: ,Ich rede von ihrer Unzucht in historicis. Nicht nur, dass den deutschen Historikern der grosse Blick für den Gang, für die Werthe der Cultur [und Kultur war für Nietzsche der Gegen-Wert zu Staat, Vf.] gänzlich abhanden gekommen ist, dass sie allesammt Hanswürste der Politik (oder der Kirche -) sind: [. . . Man muss vorerst , deutsch' sein, ,Rasse` sein, dann kann man über alle Werthe und Unwerthe in historicis entscheiden - man setzt sie fest . . . ,Deutsch' ist ein Argument, ,Deutschland, Deutschland über Alles` ein Prinzip [ . . . Es giebt eine reichsdeutsche Geschichtsschreibung, es giebt, fürchte ich, selbst eine antisemitische, - es giebt eine Hof-Geschichtsschreibung und Herr von Treitschke schämt sich nicht [...], und ich spüre die Lust, [. . .] den Deutschen einmal zu sagen, was sie Alles schon auf dem Gewissen haben. Alle grossen Cultur-Verbrechen von vier Jahrhunderten haben sie auf dem Gewissen! . . "11

Liest man dies, so erweist sich die Methode von Lukács und Hamann/Hermand nicht nur als ideologisch interessenbedingt - man schwört allenthalben auf die Stärke des Staates, der die Entfaltung des Einzelnen vermindert -, sondern diese Autoren werden selbst zu Symptomen für denjenigen Zustand, den Nietzsche schon vor hundert Jahren charakterisiert hatte.

Welche Auswirkungen nun brachte dies? bzw. welche Folgen des oben genannten Versäumnisses bestehen? Es existiert bis heute keine (publizierte) Nietzsche-Ikonografie in der Kunstwissenschaft, obwohl der Sammeltrieb doch verbreitet ist. (Das Nietzsche-Archiv in Weimar besitzt eine erste Zusammenstellung zur Nietzsche-Ikonografie. ${ }^{12}$ Die Projekte für Nietzsche-Denkmäler von Fritz Schumacher und besonders für das durch Harry Graf Kessler 1911/12 in Weimar betriebene Nietzsche-

${ }^{9}$ Heinrich Mann, Kaiserreich und Republik (1919), in: H. M. - Essays, Hamburg 1960, $408 \mathrm{ff}$., ein geradezu grundlegender Beitrag zum Verständnis der Differenz zwischen der offiziellen Ideologie des Kaiserreichs und dem Denken Nietzsches.

$10 \mathrm{Za} \mathrm{I}$, Vom neuen Götzen; KGW VI 1, 59.

Diese Passage war es übrigens, die 1919 der sozialistische Architekt Bruno Taut für sein ,Monument des neuen Gesetzes' als „,Tafel“" (sic!) plante.

$11 \mathrm{EH}$, Der Fall Wagner 2; KGW VI 3, $356 \mathrm{f}$.

12 Frau Anneliese Clauss vom Goethe-Schiller-Archiv in Weimar sei an dieser Stelle für ihre freundliche Hilfe und Kollegialität gedankt, die mir diese Nietzsche-Ikonografie 1977 zugänglich machte. 
Denkmal, das Henry van de Velde, Max Klinger und Aristide Maillol ausführen sollten, sind unbearbeitet. ${ }^{13}$

Eine Sonderung der beiden wesentlichsten Rezeptions-Stränge in der Geschichte der bildenden Kunst und Literatur, des national-panegyrischen (der über die fälschende Schwester Nietzsches und Peter Gast in den Faschismus einmündete) und des kritischemanzipatorischen, wie ich ihn nennen möchte ${ }^{14}$ (der mit den Namen Heinrich Mann, Carl Einstein, Franz Pfemfert - „Die Aktion“, Kurt Hiller - „Ziel-Jahrbücher" 1916-1920, Theodor Lessing, Albert Camus u. a. verbunden ist) ${ }^{15}$, diese Sonderung stellt eine, gegenüber den Nivellierungen von Lukács, neue Bemühung dar, die die Kunstwissenschaft insgesamt noch nicht realisiert zu haben scheint. Denn die jüngste Arbeit über die möglichen Einwirkungen auf die künstlerischen Konkretionen des frühen Max Beckmann sieht Nietzsche statt durch einen eigenen „Écran" (Zola) oder statt mit dem Bewußtsein dieser beiden Rezeptionsstränge noch durch die Brille von Lukács. ${ }^{16}$

Aber trotz dieses methodischen Einwands stellt die Arbeit von Güse eine wichtige Stufe in der sich im Fach nur zögernd anbahnenden Nietzsche-Rezeption dar. War doch der letzte Hauptbearbeiter des Euvres von Beckmann, Fr. W. Fischer, in seinen Studien zur Malerei Beckmanns dem Namen Nietzsche beinahe gänzlich ausgewichen, - ebenso zuletzt Stephan Lackner, Freund und Sammler Beckmanns ${ }^{17}$ - und das obgleich wir aus den frühen Tagebüchern wissen, daß und was Beckmann von Nietzsche las. Noch an der Front hatte er 1915 das Neue Testament und Zarathustra dabei. Übrigens war dies auch die Kriegșlektüre von Otto Dix (zu Dix später).

Eine Ausnahme innerhalb dieser desolaten Situation stellt seit Jahren der schwedische Kunsthistoriker Gösta Svenaeus dar. Er hat in seinen langjährigen Studien zur Kunst von Edvard Munch ohne Scheu und ohne Ressentiment die tiefgehenden Einflüsse Nietzsches auf Munch als Menschen und auf Munch als Maler, seine Darstellungen des nackten Menschen, der Priorität der Sonne, des "Menschenberges“ usf. erkannt und dargelegt. ${ }^{18}$ Von Svenaeus gingen folglich Impulse aus, auch in

13 Dazu die Darlegungen in den Memoiren von Henry van de Velde, Geschichte meines Lebens, München 1962; - ferner D. Schubert, Das Denkmal für die Märzgefallenen 1920 von Walter Gropius in Weimar, in: Jahrbuch der Hamburger Kunstsammlungen 21, 1976, Anm. 34.

14 Vgl. meine Besprechung von Ernst G. Güse (siehe Anm. 16), in: Zeitschrift für Kunstgeschichte, 1978, Heft 3/4, 342f. und: Expressionistische Bildnisse im Rahmen des Aktivismus, in: Die Zwanziger Jahre im Porträt - Katalog der Ausstellung Bonn 1976, 23-46.

15 Heinrich Mann 1919 (siehe Anm. 9); - Carl Einsteins Aufsätze ,,Totalität" und ,,Antike und Moderne" (C. E. - Gesammelte Werke, hg. von Ernst Nef, Wiesbaden 1962, $72 \mathrm{ff}$. und: C. E. Existenz und Ästhetik, hg. von Sibylle Penkert, Wiesbaden 1970, 47); - Franz Pfemfert, Die Deutschsprechung Nietzsches - ein Protest, in: Die Aktion, 26. Juni 1915, $320 \mathrm{ff}$. - Th. Lessing, Geschichte als Sinngebung des Sinnlosen (1916), 3. A. München 1921; - Th. Lessing, Nietzsche, Berlin 1925; - Albert Camus, Der Mensch in der Revolte, Reinbek 1951.

16 E.-G. Güse, Das Frühwerk Max Beckmanns, Bern/Frankfurt 1977

17 Friedhelm W. Fischer, Max Beckmann - Symbol und Weltbild, München 1972; - St. Lackner, Max Beckmann, Köln 1979.

18 Gösta Svenaeus, Im männlichen Gehirn,'Lund 1973; - ders., Der heilige Weg - NietzscheFermente in der Kunst Edvard Munchs, in: Munch - Probleme/Forschungen/Thesen, hg. von $\mathrm{H}$. Bock/G. Busch, München 1973, $25 \mathrm{ff}$. 
weiterer Hinsicht Nietzsche nicht aus den Augen zu verlieren: z. B. Schmoll-Eisenwerths Beitrag zur Beziehung Munchs zu Rodin (im Munch-Sammelband von 1972).

Und die Einwirkungen Nietzsches beziehen sich bei diesen Feststellungen keineswegs nur auf eine Nietzsche-Ikonografie, also nicht bloß auf die Darstellungen Nietzsches: solche gibt es von Klinger (Plastik), Hans Olde (Fotos und Radierung), ferner plastische Bildnisse von Max Kruse, Max Klein, Rudolf Saudek, Kurt Stoeving (1901) bis zu Josef Thorak 1944; - herausragt eine plastisch geniale Nietzsche-Büste vom jungen Dix, 1912 in Dresden modelliert, heute verschollen; ${ }^{19}$ herausragend ferner die malerischen Bildnisse von Munch (um 1905/06). Svenaeus hat - wie später Güse die schwierigen Fragen nach Konkretionen der Denksplitter Nietzsches in den Werken der bildenden Künstler, der Schaffenden, gestellt. Diesen Fragen widmet sich auch ein Beitrag des Unterzeichneten, der in Vorbereitung ist; dabei geht es um eine Übersicht mit Beispielen von Klinger, Peter Behrens (Vorhalle, Turin 1902), Wilhelm Lehmbruck („,Weg zur Schönheit" , ,Emporsteigender'), Munch und Beckmann um 1908/09 (Svenaeus und Güse folgend), Bernhard Hoetger (Niedersachsenstein als KriegerDenkmal), um Bruno Tauts „Monument des neuen Gesetzes" (Entwurf Dezember 1919), um Anton Hanaks Plastik, Schlemmers Tanz-Visionen, das „Licht-Gebet" von Fidus, um die Kunst von Dix in den 20er Jahren bis zu Kolbes Plastik ,Zarathustra'von 1933/34.

Desiderat und eine Studie für sich ist die Wirkung Nietzsches auf die Mäzene und die Künstler des „,neuen Weimar" (zwischen 1902 und 1912), also des Kunstzentrums, das Harry Graf Kessler entscheidend befruchtete, aufbaute und wesentlich strukturierte: Das Ziel Graf Kesslers und Henry van de Veldes (der seit 1886 Nietzsche las), einen Neuen Stil zu verwirklichen, übernational und alle Lebensbereiche einschließend, dürfte inspiriert sein von Nietzsches Kulturkritik an der Gründerzeit mit ihrem Historismus (historische Krankheit) und ihrer Stillosigkeit, die der Philosoph in seiner 1. Unzeitgemäßen 1873 radikal geübt hatte. Die Bildungs-Philisterei der Gründerzeit führe zu Pseudo-Kultur; das ,,chaotische Durcheinander aller Stile“ zu jener ,modernen Jahrmarkts-Buntheit" des Historismus. Dagegen wirkliche Kultur ist für Nietzsche „,vor allem Einheit des künstlerischen Stiles in allen Lebensäusserungen eines Volkes“. Wahre Kultur ist ihm ,,jedenfalls Einheit des Stiles“. Die Gründerzeit besitzt in seinen Augen jedoch keine Kultur, weil das „Ausschliessen und Negiren aller künstlerisch produktiven Formen und Forderungen eines wahren Stils" dominiert. ${ }^{20}$

Nun hatten ,L'Art Nouveau' in Belgien und Frankreich und der sog. ,Jugendstil in Deutschland, teils auch durch Nietzsche inspiriert ${ }^{21}$, bereits versucht, alle Lebens-

19 Die Nietzsche-Büste von Dix bei Paul F. Schmidt, Otto Dix, Köln 1923 gewürdigt; - in: Kunst der Nation, Oktober 1934: Nietzsche-Bildnisse (von F. Paul = Paul F. Schmidt, der von den Nazis entlassene Direktor des Stadtmuseums Dresden); - Maria Wetzel, Ein harter Mann dieser Maler - Interview mit Otto Dix, in: Diplomatischer Kurier, 14. Jg. Köln 1965, S. 734 und abgebildet und besprochen bei D. Schubert, Otto Dix - in Selbstzeugnissen und Bilddokumenten, Reinbek 1980.

20 UB, DS 2; KGW III 1, 159, 161 f. - Zugleich entwarf Nietzsche den Plan zu ,Die Möglichkeit einer deutschen Cultur".

21 Fr. Ahlers-Hestermann, Stilwende, Berlin 1941, 87, 120; - Dolf Sternberger, Uber den Jugendstil, Hamburg 1956, 42 ff. - Robert Schmutzler, Art Nouveau - Jugendstil (1962), Stuttgart 1977. 
bereiche zu umgreifen. Doch wurde erst der Aufschwung der Künste zu einem neuen Stil, den Van de Velde und Kessler mit anderen Künstlern bewirkten, zu solch einer breiten Bewegung, die das erreichen wollte, was Nietzsche als ideelle und produktive Einheit des künstlerischen Stils verstand und forderte, als er die Gründerzeit-Kultur als Bildungs-Philisterei verdammte.

Dabei muß man sich bewußt halten, daß Van de Velde für seine Kunst das Etikett ,Jugendstil' abgelehnt hat; tatsächlich gehen seine Kunst und Kesslers Ziele weit über den Jugendstil hinaus, nicht zuletzt aufgrund der Ideen Nietzsches. ${ }^{22}$ Das Anliegen war: Weimar als Zentrum nicht der nationalistischen (antisemitischen, frankreichfeindlichen) Kunst, sondern der neuen, übernationalen, europäischen Avantgarde des Belgiers Van de Velde, Munchs (in Weimar 1906 - Ausstellung des deutschen Künstlerbundes, wo er mit Beckmann zusammentreffen konnte), die Förderung des Franzosen Aristide Maillol durch Kessler seit 1906, die Rodin-Ausstellungen Kesslers in Weimar 1904 und 1906, der Plan für ein monumentales Nietzsche-Denkmal dort, das nicht zustande kam; das Abbe-Denkmal in Jena (von H. van de Velde, Reliefs von Meunier, Büste von Klinger, 1911 vollendet). In diesen Hinsichten ist - zumindest was den panegyrisch-nationalistischen Nietzsche-Strang anbelangt - Neues zu erwarten in der Berliner Dissertation von Jürgen Krause. über den Weimarer Nietzsche. ${ }^{23}$

Daß selbst ein als kritisch eingestufter Elends- und Kriegs-Maler und Grafiker wie Otto Dix, der in der Geschichtsschreibung der DDR als Paradigma gilt und ideologisch in Dienst genommen ist, kein Marxist sondern Nietzscheaner war, wird überraschen. Dix ist durch Fritz Löffler bearbeitet; die Monografie erschien jüngst in 4. Auflage (Dresden 1977). Daß Nietzsche darin jedoch nur spärlich erwähnt ist, nur quasi äußerlich, darf man nicht allein dem verdienstvollen Autor anlasten.

Dix las jahrelang immer wieder im Zarathustra und vor allem in der Fröblichen Wissenschaft. Zu seiner Beziehung zu Nietzsche bekannte er sich noch 1965 in einem Interview. ${ }^{24}$ Dix modellierte nicht nur jene vehemente Nietzsche-Büste (ca. $60 \mathrm{~cm}$ hoch, Gips, dunkelgrün bemalt), die die Nazis aus dem Dresdner Stadtmuseum entfernten, ${ }^{25}$ sondern hat in seiner Malerei die „Anbetung des Scheins“, die der späte Nietzsche „Schein" anders als 1872 - von den Schaffenden forderte (FW, Vorrede zur 2. Ausg. 1886), vollzogen und damit eine grundlegend neue Ästhetik gegenüber dem 19. Jahrhundert und dem Expressionismus realisiert. Dix malte das, was er mit Augen sah. Er gestaltete im „Verlangen nach dem Häßlichen“, im Willen zum „Bilde alles Furchtbaren, Bösen, Rätselhaften, Vernichtenden, Verhängnisvollen auf dem Grunde des Daseins", ja mit einer "Tapferkeit des schärfsten Blicks, die nach dem Furchtbaren

22 Henry van de Velde, Kunstgewerbliche Laienpredigten, Leipzig 1902; - ders., Zum neuen Stil, München 1955.

Wichtig sind von Kessler die frühen Tagebücher und Briefe an H. van de Velde (im Nachlaß in Marbach und Zürich, Van-de-Velde-Nachlaß), die auszuwerten wären.

23 Jürgen Krause, Der Weimarer Nietzsche, phil. Diss. bei Tilman Buddensieg, FU Berlin.

24 Dix im Interview mit Maria Wetzel 1965 (s. Anm. 19), S. 740.

25 Siehe Anm. 19. - Der mit Dix befreundete Fotograf Hugo Erfurth hatte die NietzscheBüste von Dix fotografiert, bevor sie 1937 von den Nazis entfernt und auf die Auktion der Galerie Fischer (Luzern) im Jahre 1939 gegeben wurde; seitdem ist sie verloren. Alte Fotos befinden sich im Besitz von Frau Martha Dix, Hemmenhofen, der ich für Auskünfte vielmals danke. 
verlangt, [...] an dem sie ihre Kraft erproben kann" (GT, Versuch einer Selbstkritik 4 und 1).

Der Realismus von Dix - eine ,dionysische` Malerei? Das wäre zu bejahen insofern, als das dionysische Prinzip nicht die bloße Entfesselung des Erotischen und Sinnlichen (Rausch) bedeutet, ${ }^{26}$ sondern in Nietzsches umfassenderem Verständnis zugleich den tragischen „Urschmerz" des Daseins, den Wandel (mit Heraklit), die Verwandlung und Zerstörung, den Wahnsinn, Tod und mythische Wiederauferstehung miteinschließt. ${ }^{27}$

Nicht nur beinahe alle Dichter und Literaten im frühen 20. Jahrhundert, auch beinahe alle bildenden Künstler haben Einflüsse der Denksplitter Nietzsches aufgefangen und umgesetzt, Konkretionen, die zu untersuchen sich lohnen würde, die für das Verständnis der Ideen und Gestaltungsprinzipien von Lebenskult-Kultur, Symbolismus, Werkbund-Zeit, Futurismus und Expressionismus unverzichtbar sind. In der Germanistik haben W. Taraba und Peter Pütz schon 1963, Gunter Martens 1971, H. G. Kemper 1974, Silvio Vietta und B. Hillebrand in jüngster Zeit die Fragen nach den Einflüssen Nietzsches auf die Inhalte und Formen der Dichtung um und nach 1900 gestellt und $\mathrm{zu}$ beantworten begonnen. ${ }^{28}$ In der Kunstgeschichtswissenschaft geht freilich die Möglichkeit, einen führenden Rang unter den Geschichtswissenschaften einzunehmen, eher verloren, wenn sie in einer solch zentralen Frage zurückbleiben sollte. Dies gilt sowohl für die konservativen Positionen, die sich der kritischen Untersuchung der ideengeschichtlichen Bezüge verschließen, für die Sachverwalter der „Stilgeschichte" und der ,Ikonografie", die die Einheit der Kunstwerke - die Hegelsche Einheit von Gestalt und Bedeutung - sinnentstellend teilen, als auch für die

${ }^{26}$ Das umfassendere Verständnis von ,dionysisch` ist $\mathrm{z}$. B. Henning Müller entgegenzuhalten im Hinblick auf die 1848 entstandene Marmorskulptur von Kalide, „Bacchantin auf einem Panther", von der Müller sagt, sie sei ,,wahrscheinlich das erste dionysische Kunstwerk in Deutschland überhaupt. In seiner Aussage wird es allenfalls von Nietzsches Philosophie übertroffen ..." $(\mathrm{H}$. Müller, Denkmalstil und Menschenbild, in: Jahrbuch Preussischer Kulturbesitz, 14, 1977, Berlin 1979, S. 255). Noch weniger kann ich Müllers Vergleich zwischen „Pan tröstet Psyche" von R. Begas (1856) und Nietzsches Philosophie, der als eine gleichwertige Parallele qualitativ durchgeführt ist, zustimmen. Die Begas'sche Plastik steht zu sehr im offiziellen Kunstgeschmack der preußischen Zeit, als daß sie eine Umsetzung von Nietzsches Ideen auch nur annähernd - besonders 1856 - sein könnte. Weit eher wäre - wenn überhaupt für die Bildnerei (die dem Nietzsche von 1872 appollinisch war!) - angesichts der Plastik Rodins von einer herakliteischen Kunst zu sprechen (wie das schon Georg Simmel tat), die dionysische Dimensionen hat. - Zu Simmel und Rodin vgl. J. A. Schmoll gen. Eisenwerth, in: Ästhetik und Soziologie um die Jahrhundertwende - Georg Simmel, Frankfurt/M. 1976, 18-43 (jedoch ohne die Nietzsche-Frage zu stellen).

27 D. Jähnig, Nietzsches Kunstbegriff, a. a. O. 1972, $47 \mathrm{f}$.

28 Wolfgang Taraba, Der schöpferische Einzelne und die Gesellschaft in Nietzsches ,Zarathustra", in: Literatur und Gesellschaft - Festgabe für Benno von Wiese, hg. von H. J. Schrimpf, Bonn 1963, $196 \mathrm{ff}$. - Peter Pütz, Kunst und Künstlerexistenz bei Nietzsche und Thomas Mann - zum Problem des ästhetischen Perspektivismus in der Moderne, Bonn 1963; - Bruno Hillebrand, Artistik und Auftrag - zur Kunsttheorie von Benn und Nietzsche, 1966; - Gunter Martens, Vitalismus und Expressionismus, Stuttgart 1971; - H. G. Kemper, Vom Expressionismus zum Dadaismus, Kronberg 1974; - S. Vietta/H. G. Kemper, Expressionismus, München 1975, $134 \mathrm{ff}$. und B. Hillebrand ( $\mathrm{Hg}$.) Nietzsche und die deutsche Literatur, 2 Bände, München 1978. 
,progressiven' Strömungen der sozial-kritisch Orientierten und der Ideologiekritik, wenn sie die Bedeutung Nietzsches gar nicht oder nur durch die Vordeformierung bei Mehring, Lukács oder S. F. Oduev ${ }^{29}$ sehen wollen. Zum Beispiel spielt in der seit Jahren währenden Historismus-Diskussion ${ }^{30}$ wiederum Nietzsche theoretisch nicht die ihm gebührende Rolle, obwohl er 1874 mit seiner 2. Unzeitgemäßen Betrachtung („Vom Nutzen und Nachteil der Historie für das Leben“) die ,,kritische Art der Historie" begründet und zugleich eine grundlegende Kritik der ,, historischen Krankheit" seiner Epoche (der Zeit nach dem Sieg von 1871, der ihm eine „Exstirpation des deutschen Geistes zugunsten des ,deutschen Reiches“" war) geliefert hatte. Er lehnte eine Geschichtsauffassung allein ,,vom Standpuncte des Erfolges" und den historischen Optimismus seiner Zeit ab: ,Wer nicht begreift, wie brutal und sinnlos die Geschichte ist, der wird auch den Antrieb gar nicht verstehn, die Geschichte sinnvoll zu machen." (Entwürfe zu Wir Philologen 1875; KGW IV 1, 5[58], 133) ${ }^{31}$

Ebenso wäre es auf einem anderen Forschungsfeld, der Kunst des von der französischen Avantgarde ideengeschichtlich wesentlich verschiedenen Expressionismus, dringend an der Zeit, wieder und wieder Fragen nach Nietzsche-Konkretionen aufzuwerfen. Dies ginge von einer schwierigen Metaphorik bis zu einem in aller Munde befindlichen Begriff wie dem der ,Brücke“-Künstler (Dresden 1905), die zu den Protagonisten des Expressionismus (der Malerei) gehören. Der Titel , Brücke“ für den Zusammenschluß der jungen Schaffenden 1905 dürfte mit Sicherheit eine Entlehnung aus Zarathustra sein; wichtiger, daß die Ideen des Vitalismus, die Spannungen zwischen einem (von Nietzsche und Simmel kommenden) radikalen Individualismus und dem "Gemeinschaftsgefühl" des Expressionismus, einen neuen Menschen und eine neue Zeit heraufzuführen, und vor allem die weit gefächerte Lichtsymbolik in Wort und Bildern auf die Passagen im Zarathustra zurückzuführen sind (Sonne- und Sternsymbolik Zarathustras). Während Kunsthistoriker jener Jahre des Aufbruchs und der Kunstrevolution wie Fritz Burger, G. F. Hartlaub und Eckart von Sydow ihre Bücher und Beiträge ${ }^{32}$ im vollen Bewußtsein der enormen Wirkung Nietzsches auf diese Kunstrevolution des Expressionismus schrieben, erwähnen die heutigen Bücher,

29 S. F. Oduev, Auf den Spuren Zarathustras (Moskau 1971), Berlin-Ost 1977.

30 Michael Brix/Monika Steinhauser, ,Geschichte allein ist zeitgemäß" - Historismus in Deutschland, anabas-Verlag. Lahn-Gießen 1978, - darin H. Dilly: Entstehung und Geschichte des Begriffs ,Historismus', S. 11-16. - Zu Nietzsches Historismus-Kritik vgl. dagegen schon Jürgen Habermas, Zu Nietzches Erkenntnistheorie - ein Nachwort (1968), in: Kultur und Kritik, Frankf./M. 1973, $244 \mathrm{f}$.

31 Zur Bedeutung von Nietzsches 2. Unzeitgemäßer und von „Wir Philologen“ vgl. Th. Lessing, Geschichte als Sinngebung des Sinnlosen, 1916; - Ernst Langlotz, Uber das Interpretieren griechischer Plastik, Bonner Universitätsreden, Heft 7, Bonn 1947, 8f. - D. Jähnig, Nietzsches Kritik der historischen Wissenschaften, in: Praxis, 6, 1970, $223 \mathrm{f}$. und ferner im Úberblick Reinhart Koselleck, Geschichte - Historie, in: Geschichtliche Grundbegriffe, hg. von O. Brunner/W. Conze/R. Koselleck, Bd. 2, Stuttgart 1975, 701-02 und 706f.

32 Fritz Burger, Einführung in die moderne Kunst, Berlin 1917; - E. von Sydow, Das religiöse Bewußtsein des Expressionismus, und: Der doppelte Ursprung des Expressionismus, beide in: Neue Blätter für Kunst und Dichtung (Dresden), 1. Jg. 1919, $193 \mathrm{ff}$. und $227 \mathrm{ff}$. - ferner derselbe, Die deutsche expressionistische Kultur und Malerei, Berlin 1920; - G. F. Hartlaub, Kunst und Religion, Leipzig 1919. 
die ,Standardwerke' sein wollen, den Namen des ,Flammenwerfers" Nietzsche entweder nur für subalterne Fragestellungen oder aber überhaupt nicht. ${ }^{33}$

Der Einfluß und die Einwirkungen Nietzsches gehen über seine ,Ikonografie' und über die Projekte für seine (nicht errichteten) Denkmäler weit hinaus in die Ideen der Zeit und somit in die Dichtungen, in die symbiotischen Freundschaften der Künstler und der Künstlergruppen damals, in die Formen und die Gehalte der Grafik, der Malerei, der Plastik und selbst der Baukunst. Wenn in der Kunsthistorik Nietzsche nicht mehr jene ungelesene oder dem Präfaschismus verdächtige Gestalt sein sollte, wird auch die Geschichte der Kunst des 20. Jahrhunderts anders geschrieben werden als bislang. Denn es ist mit Nietzsche, ,gar nicht abzusehen, was alles einmal noch Geschichte sein wird. Die Vergangenheit ist vielleicht immer noch wesentlich unentdeckt! Es bedarf noch so vieler rückwirkender Kräfte!“ („Historia abscondi$\left.\mathrm{ta}^{\mathrm{c}}\right) \cdot{ }^{34}$

33 Hans Sedlmayr, Die Revolution der modernen Kunst, Hamburg 1955; - Wolfgang Hütt, Deutsche Malerei und Grafik im 20. Jh., Berlin-Ost 1969; - Paul Vogt, Geschichte der deutschen Malerei im 20. Jh., Köln 1972; - Peter Selz, German Expressionist Painting, Berkeley 1957, 2. A. 1968; - R. Hamann/J. Hermand, Expressionismus, Berlin-Ost/ München 1976.

${ }^{34} \mathrm{FW}, 1$. Buch, No. 34. 